\title{
Traditional Chinese Medicine Physicians' Insights into Inter-Professional Tensions between Traditional Chinese Medicine and Biomedicine: A Critical Perspective
}

\author{
Leanne Chang, $\mathrm{PhD}$ \\ School of International Communications \\ University of Nottingham Ningbo China \\ Ningbo 315100, China \\ Email: Leanne.chang@nottingham.edu.cn
}

\author{
Jing Ci Jill Lim, BA with Honours \\ Department of Communications \& New Media \\ National University of Singapore
}

Singapore 117416

Acknowledgment: This research was supported in part by the Humanities and Social Sciences Seed Fund, Office of the Deputy President, National University of Singapore. 


\begin{abstract}
In Singapore, the institutional preference for biomedicine and the cultural importance of traditional Chinese medicine (TCM) have created tensions between the two medical systems and erected barriers to a more collaborative healthcare system. This study foregrounds TCM physicians' voice to reveal ideological struggles and power imbalances that underlie the interprofessional tensions and accompanying marginalization of TCM. Through in-depth interviews with 22 TCM physicians in Singapore, this study reveals the incongruences in ideological underpinnings between biomedicine and TCM, reflected in their different worldviews and epistemological approaches to knowledge formation and evaluation. Power differentials between the two medical systems are manifest in TCM physicians' inferior position in relation to their biomedical peers, the patients' internalization of biomedical standards to question the TCM profession and their own interest in seeking TCM treatments, and the state's limited support for TCM research, subsidies, and service provision in hospital settings. The results suggest that more open dialogue about the dichotomous framings of biomedicine and TCM is key to disrupting the mutual reinforcement of ideology and power, as well as to creating increased mutual understanding between the two medical systems.
\end{abstract}

Keywords: discourse; ideology; integrative medicine; medical pluralism; power; traditional Chinese medicine 
The integration of biomedicine and traditional medicines has become a health system goal in many countries (World Health Organization, 2008). In Singapore, biomedicine is institutionalized as the orthodox medical practice in healthcare and medical education systems (Lee, 2006; Quah, 2003). Concurrently, the use of traditional modalities such as Ayurveda, traditional Chinese medicine (TCM), and Malay medicine is common among ordinary people, given the country's cultural and racial diversity (Lim, Sadarangani, Chan, \& Heng, 2005). Among the various traditional healing modalities, TCM appeals to the majority Chinese population and is perceived as important in Chinese heritage in Singapore (Ministry of Health Singapore, 2010). Empirical data indicate that the majority of Chinese Singaporeans have sought TCM treatments for themselves or their family members (Koh, $\mathrm{Ng}, \&$ Teo, 2004; Lim et al., 2005). The cultural context of Singapore provides a venue for medical pluralism but also identifies a need to examine whether the pursuit of integrative medicine offers fair recognition of different healing knowledges.

In the wave of modernization and globalization, biomedicine has become the dominant paradigm in many pluralistic medical systems, including those where indigenous therapeutic practices continue to be an inseparable part of everyday life (Airhihenbuwa, 1995). The controversy, however, is that the new global discourse has created a separation between biomedicine and the others (Dutta, 2008; Schreiber, 2005). Inasmuch as biomedicine has become the orthodox knowledge system, traditional medicines are framed as alternative, fringe, primitive, unscientific, and, ironically, unconventional. The dichotomous distinction of the orthodox and unorthodox often leads to distrust in and disapproval of traditional knowledge and practices, which in turn reinforces the privileged position of biomedicine and the subordination of traditional medicines (Khan, 2006, 2014; Lambert, 2012). 
In the context of Singapore, while a few studies have explored consumers' responses to the asymmetric healthcare system (Chang \& Basnyat, 2015; Ku, Tan, \& Li, 2001), little research has examined the marginalization of TCM from TCM practitioners' perspectives. This study is intended to explore TCM practitioners' interpretations of inter-professional tensions and the underlying mechanisms that result in power imbalances. Research like this is important because TCM and biomedical physicians need to increase their interactions in light of the prevalence of TCM in Singapore and establish a more collaborative healthcare system in which both parties cooperate to provide patients with better service. To achieve these goals, it is necessary to articulate power relations between the dominant and subordinate groups and to address the interprofessional dynamics from TCM physicians' perspectives.

\section{A Critical Social Science Perspective}

Critical communication scholars have highlighted the importance of inquiring into the relationship between power and control to offer alternative ways of researching health communication (Zoller \& Kline, 2008). For instance, Airhihenbuwa (1995) illustrated the use of a post-colonial approach to analyzing the perpetuation of allopathic hegemony in healthcare and epistemological systems and the resulting marginalization of traditional medicines. Lupton (1994) proposed the use of a social constructionist approach to examine the dominant framings of health and the ideological dimensions of language in medical discourse to disrupt power differentials between dominant and subordinate groups. Dutta (2008) introduced the culturecentered approach, which emphasizes revealing the status quo embedded in structures and critically interrogating subalternity through praxis of agency and communicative acts. Researchers like Basnyat (2017), Basu and Dutta-Bergman (2007), and Khan (2006, 2014) have also brought forth issues of culture, ideology, and disparities to reveal tensions between tradition 
and modernity, and challenge taken-for-granted assumptions in biomedical practice.

In response to the field's call for more critical inquiries into inequalities in health (Lupton, 1994), this study uses a critical social science perspective (CSSP) to explore ideological conflicts and power relations between TCM and biomedicine within TCM physicians' day-today practice (Eakin, Robertson, Poland, Coburn, \& Edwards, 1996). Drawing on critical sociology theories, CSSP holds that the provision of healthcare services is shaped by social and structural processes, which involve the domination of one group over another, the exposing of power imbalances, and the marginalization of the other (Adams, Hollenberg, Lui, \& Broom, 2009). CSSP emphasizes ideology and power as key theoretical constructs. Ideology amalgamates a group of beliefs, customs, practices, and behaviors that represent social reality. Ideology can be infused into the repository of truth and becomes a normative tool for hegemonic control (Filc, 2004). Here, hegemony refers to control through moral and intellectual leadership without the use of coercive force (Elling, 1981). Power, in a neutral sense, can be understood as the ability to make decisions and maintain control (Lukes, 2005). From a critical perspective, however, power demonstrates an individual or group's ability to set and influence agendas through which potential conflicts are excluded or suppressed. Power can also reflect one's ability to influence others such that they act against their own interests (Adams et al., 2009). CSSP holds that the macro-level influences of ideology and power can shape expectations and interactions between health professionals and patients and among health professionals of different medical systems. Thus, it is important to make certain dominant aspects of ideology and the exercise of power overt so that they can be questioned (Eakin et al., 1996). With a focus on ideology and power, CSSP is useful in uncovering inclusionary and exclusionary strategies employed to establish epistemic authority and maintain supremacy (Keshet \& Giveon, 2013). 


\section{Ideological Differences Between Biomedicine and TCM}

Biomedicine is an extension of Euroscience, which emphasizes the use of scientific rules and tools to uncover truth (Barry, 2006). This medical system, however, has been critiqued for its ideology in reductionism, only responding to "observable pathology" in the form of "organs, tissues and body fluids" (Russo, Ania, \& Crellin, 2012, p. 54). The reductionist approach tends toward outlining abnormalities in one's biological makeup as the cause of illness, in turn excluding the wider factors that can affect one's well-being (Lupton, 2012). Critics of biomedicine also assert that biomedical doctors adopt a materialistic ideology and rely on a pattern of relationship in which the doctors dominate the interaction instead of allowing for mutual participation as seen in a patient-centered relationship (Dutta, 2008).

In contrast, TCM is built on a holistic ideology that focuses on balance within a person and between the person and the environment $(\mathrm{Hu}, 2016)$. This holistic worldview is similar to many other traditional practices worldwide (Airhihenbuwa, 1995). TCM holds that good health originates from optimal interconnections among a person's physical, spiritual, and mental states. For instance, TCM works around five organs -heart, liver, spleen, lung, and kidney, all of which are connected by Qi and blood. It is crucial to keep all interconnected structures functioning harmoniously. The theory of yin and yang is deeply rooted in the TCM system and used to direct clinical diagnosis and treatment. The emphasis on harmony between the universe and a person's internal system distinguishes TCM from biomedicine's focus on external reality. However, the different ways of knowing and reasoning have also led to a perception that TCM is based on a mythology of nature and is not scientific (Schreiber, 2005).

Critical scholars argue that the ideological dominance of biomedicine has resulted in a devaluation of other knowledge systems (Airhihenbuwa, 1995; Dutta, 2008). The effect of 
subjugation creates a biomedical "flatland," an epistemology that only accepts what is considered scientific and objective based on biomedical standards (Hollenberg \& Muzzin, 2010, p. 39). The strong foothold of biomedicine over other healing systems has also spiraled into a global type of medical dominance, excluding contributions to new knowledge from other medical paradigms (Russo et al., 2012). The ideological struggles that TCM physicians face in a biomedicine-dominant health system is one focus of the present study.

\section{Power Dynamics in Healthcare}

Past research has examined power relations in healthcare with respect to doctor-patient communication and interactions among healthcare professionals (Lupton, 2012). In doctorpatient communication, power is present in a biomedical doctor's capacity to detain patients and require them to undergo therapy. With control of knowledge, biomedical doctors are able to maintain a hegemonic status and use their authority to define health and illness and to demand patient compliance. Conversely, traditional practitioners cannot exert power at the same level (Wiese, Oster, \& Pincombe, 2010). The contrast alludes to the subordinate status of traditional medicine and its lack of legitimacy in biomedical-dominant systems (Lupton, 2012).

At the inter-professional level, Hollenberg (2006) noted that biomedical doctors exercise dominance through controlling patient referrals, delegating traditional practitioners to performing basic tasks within specified parameters, and using biomedical language in their communication. In this way, traditional practitioners can only obtain biomedical peers' recognition based on their technical value. An example is the wide practice of acupuncture outside the TCM paradigm in North America, the United Kingdom, and Europe. In most of these settings, acupuncture is reduced to biomedical logic and its knowledge base is excluded from discussion (Hollenberg \& Muzzin, 2010). In response to the power imbalances, some traditional practitioners resort to a 
“deferential strategy," in which they accept an inferior position in relation to biomedicine for inclusion in the mainstream healthcare system (Wiese et al., 2010, p. 337). Others strive to gain external legitimacy to garner state recognition for traditional practices, ensure professional boundaries around their practice, and protect their occupational autonomy (Dutta, 2008).

Extant research on integrative medicine suggests that tensions exist between biomedicine and TCM, arising from different paradigmatic worldviews and different approaches to healing. Power and resources given to different medical systems can shape patterns of service provision and reinforce unequal statuses within a country (Hollenberg \& Muzzin, 2010). This may cause TCM and biomedical professionals, who are supposed to work together to provide patients with the best possible medical treatment, to remain distant.

\section{Traditional Chinese Medicine in Singapore}

Since Singapore gained independence, the government has taken a "laissez-faire" approach to controlling TCM, based on the belief that TCM is part of the cultural heritage (Quah, 2003, p. 2005). After the rapid growth of China, Singapore's government shifted to active scrutiny and regulation of TCM in response to the revival of TCM, the increasing worldwide interest in holistic medicine, and the state's mission to build a multifaceted medical hub in the region (Lee, 2006). Health authorities in Singapore recognize the importance of TCM and have initiated efforts to ensure its safe practice. In November 2000, the Parliament passed the TCM Practitioners Act, which aims to strengthen the professional standards of TCM practitioners. Anyone who practices TCM in Singapore, including prescribing herbal medicine and carrying out heat therapy, massage, and acupuncture, is required to register with the TCM Practitioners' Board and possess a valid practicing certificate (Ministry of Health Singapore, 2014). As a result, by 2005, most TCM practitioners were diploma holders from three training institutions: 
Singapore College of TCM, Chung Hwa Medical Institution, and Nanyang Technological University of Singapore (NTU), which collaborated with Beijing University of Chinese Medicine on a five-year double degree course in TCM and biomedical science (Lee, 2006).

Although TCM has achieved partial autonomy via state intervention, biomedicine remains the dominant model in Singapore. The institutional preference for biomedicine over TCM to some extent reflects the state's responses to the globalization of Western scientific worldviews and its endeavors to take part in global medical discourse (Lee, 2006; Lim et al., 2005). With these political conditions in the background, this study foregrounds TCM physicians' voice and seeks to explore how they observe tensions between biomedicine and TCM within the framework of ideology and power. Specifically, we raised the following research question: How do TCM physicians interpret the ideological differences and power dynamics between biomedicine and TCM embedded in their day-to-day practice?

\section{Method}

\section{Participants and Data Collection}

The criteria for participation were TCM physicians who are registered with the TCM Practitioners' Board and possess a valid practicing certificate. This study aimed to sample a pool of participants reflecting diverse backgrounds in age, gender, and years of experience. Using purposive sampling strategies, participants were recruited from various TCM institutions, including private TCM clinics and TCM departments housed within public hospitals and universities. Email invitations were sent to 35 TCM institutions in Singapore. Interested physicians could reply to confirm participation and administrative arrangements were made to schedule an appropriate interview day and time. Of the 30 participants approached, 22 TCM physicians between the ages of 29 and 80 responded and were subsequently interviewed $(M=$ 
47.32, $S D=14.64)$. The location and time of interview were decided based on participants' convenience. Omitting socio-demographic criteria for inclusion led to a natural selection of participants from different demographics and backgrounds. Twelve participants were males and 10 were females. Eight had less than 10 years of experience in TCM, nine had between 10 and 29 years, and five had between 30 and 50 years. Eleven had a minimum diploma or certificate in TCM, eight had a bachelor's degree in Chinese medicine, and three possessed a post-graduate degree. Most participants were from private TCM clinics, while two were from the National University Hospital Acupuncture clinic, two were engaged in home practice, and one was from the NTU Chinese Medicine Clinic.

The National University of Singapore Institutional Review Board approved the interview study. Verbal consent was obtained from participants prior to the interview. Participants were informed of the purpose of the study, the recruitment criteria, the interview process, sample interview questions, and how their data would be used and protected. The semi-structured interview guide covered areas such as participants' perceptions of the dynamics between biomedicine and TCM, tensions observed in medical principles and theoretical foundations, the extent of institutional recognition and government support for TCM, experiences of any status or power imbalances, and interactions with patients concerning biomedical use. Each participant received SGD \$30 supermarket vouchers ( USD\$22) for their contributions to the research. Although all participants completed the interview, they were informed that they could withdraw from the study any time during the interview and keep the incentive. The second author conducted all interviews between January and February 2015. Eighteen interviews were conducted in Chinese and four in English. Local undergraduate researchers transcribed the interviews verbatim in both Chinese and English. Then the two authors translated all Chinese 
transcripts into English independently and crosschecked the translation results. Each interview lasted approximately 60 minutes, resulting in 22 taped hours and 408 pages of text.

\section{Data Analysis}

Thematic analysis was used to identify salient themes in the interview data (Braun \& Clarke, 2013). With the research question in mind, both authors started by reading and rereading the transcripts to familiarize with the data. Following up with data coding, we highlighted words, phrases, and sentences carrying manifest or latent meanings relevant to our theoretical framework. We then identified similarities and overlaps among the initial codes and clustered them into candidate themes. Through sorting, re-sorting, and reiterative discussion, we examined each candidate theme's ability to cover the collated extracts and capture their essential meanings in relation to our research question. Results of thematic analysis were the emergence of three meta-themes, each organized participant responses into an internally consistent and theoretically meaningful account.

\section{Results}

Three meta-themes emerged from the data that summarized participant accounts of the ideological differences and power relations between biomedicine and TCM. The first metatheme revealed the confrontation of knowledge systems infused with ideological struggles. The second meta-theme presented power differentials lurking in TCM physicians' interactions with biomedical doctors and with patients. The third meta-theme addressed how political forces reinforced allopathic hegemony through the allocation of research resources, the provision of subsidies, and the granting of decision-making authority in hospital settings.

\section{Struggle Over Different Ways of Knowing}

Participants held that knowledge development in TCM is scientific in its own way, i.e., 
that knowledge creation and verification involve a systemic experimentation process rooted in trial and error, as well as years of observation. This experience-based epistemology derives a body of knowledge and nomenclature that differ from the biomedical system, where positivist thinking, replicable verification, and standardization are rules for ascertaining knowledge claims. However, divergences in the two knowledge systems have created barriers to communication, as well as judgments of which approach is valid:

In TCM, we talk about five elements - metal, wood, water, fire, and earth. Western doctors do not have such concepts, unless they have deep knowledge of the Chinese cultural and educational backgrounds. Many people feel that TCM is weird, just because it is knowledge passed down across generations. But in fact it is based on years of experience by the previous generation of TCM physicians, trying different medicinal herbs on different patients.

Ideological struggles are present in two forms in this example. One is the struggle over the legitimacy of TCM's use of non-biomedical language to develop knowledge. The discursive spaces are supportive of biomedical epistemology such that TCM physicians are under pressure to use biomedical language to make themselves understandable. The other struggle is over conflicts regarding what is considered an adequate mode of learning. TCM physicians are trained based on experiential learning in which knowledge is articulated, circulated, and verified through test and retest procedures across generations. This learning mode is systematic in its own right, but it has received criticism for being old-fashioned and lacking rigor from a Western scientific perspective. Participants noted that TCM's appreciation of old ideas does not satisfy the biomedical emphasis on continuous progress and advances in innovations:

There are articles found in TCM classics. The older the classic is, the more value it has. 
For example, things like what the ancestors have said, how this medicine should be taken. For biomedicine, they must use the newest, latest things in order to have persuasive power. Because to them, old things are considered outdated.

Participants stated that TCM has been perceived as outdated and lacking improvement, but the value of TCM lies exactly in its thousand-year history. Here, ideological differences reside in conflicting worldviews, such as the meanings of old and new. Participants saw positive meanings in "old," such as being "valuable," "reliable," and "persuasive." However, they felt that these meanings only occupy a marginal place in the mainstream discourse because they do not coincide with the biomedical focus on progress and innovation.

Similarly, ideological struggles appear in TCM's reliance on experiential knowledge visà-vis biomedicine's emphasis on positivist evidence. Western scientific methods are the foundation of biomedical training and the benchmark for assessing the reliability and effect of biomedical treatments. Biomedicine requires the same level of standardization from TCM, which participants found hard to follow. Pulse reading is one example:

For biomedicine it is very simple, they look at your resting heartbeat. But it is different for TCM. There are 28 different pulses in TCM. Pulse reading is very subjective, different people doing pulse reading on the same person can have different findings. So how are you going to convert something that is very subjective into science?

TCM physicians rely on an abstract thinking style to make inferences based on observed symptoms. Participants' bitter tone revealed the powerless feeling originating from the inability to convert TCM knowledge into biomedical nomenclature. To defend the discipline, TCM physicians have to meet the requirement of standardization. This very process of subjecting TCM to the scrutiny of the biomedical model, however, reproduces the inferiority of TCM. 
Scientific data and tools establish grounds for the objectification of biomedical practice. Differing from such sub-atomic makeup of the physical universe, TCM focuses on correcting imbalances in the body and solving the root problems such that treatments are tailor-made according to each patient's physical constitution and medical condition. Participants found it difficult to reconcile their reasoning system with biomedicine, which stresses a standard treatment formula and the match of prescriptions with a fixed set of symptoms. Holism guides through the diagnostic process and the prescription of drugs in TCM. As such, participants noted that TCM research is usually constrained by the lack of a fixed system in place:

In TCM physicians prescribe medicine based on the patient's pulse diagnosis, tongue reading, etc. In TCM it is very difficult to do research because for research you need to have very definite amounts. For example, how many grams of this medicine? But TCM is different. The amount of medicine you give varies according to how the body changes. It depends on how experienced the physician is. How are you going to do research if it's based on the physician's experience?

Participants felt that they have no capacity to go against biomedical diagnoses. Since biomedical doctors rely on medical equipment and lab tests to produce a diagnosis, everything is laid down objectively in black and white and there is no room for argument:

Western doctors usually rely on medical equipment to produce an accurate diagnosis. If you talk about contesting or going against them, in what capacity can we do so? We can only rationalize using our four methods of diagnosis, “Wangwenwenqie” (望闻问切), which means looking, listening, asking, and feeling the patient's pulse. In this case, we don't really have much of an advantage.

The perpetuation of the biomedical model's technological imperatives and its universality 
made participants doubt whether TCM's holistic and personalized approach is valueless when confronting biomedicine's equipment-based diagnostic approach, which treats patients as atomized units. Additionally, allopathic hegemony created a norm perception that medical instruments and technologies are exclusive to biomedical use. TCM physicians are restricted in their rights to use medical equipment to assist in diagnosis and have no choice but to refer patients to biomedicine. In this way, biomedicine has an advantage afforded by the technology that propels its progress while limiting the growth of TCM in the healthcare industry:

Under some situations, you have to make recommendation, asking the patient to go in for a checkup using modern equipment. But the thing is that MRI and CT scans are not exclusive for biomedicine's usage. In China, many TCM hospitals have that equipment. So long as you are a doctor you can use it. Many people have the wrong impression that MRI belongs to biomedicine's patent. But that's not true.

The interview data reveal sites of ideological struggles where differences exist in the two medical systems' epistemologies, learning modes, and ways to define and verify evidence. The dominant paradigm is able to normalize its perspectives through framing the TCM way of reasoning as other and demoting its legitimacy in discursive spaces. The dichotomous separation of healing knowledges extends to a distinction between which group is entitled to use technology and which is not. While TCM is devalued for its lack of Western scientific backing, depriving TCM physicians of the right to use equipment further diminishes their chance to develop the discipline in their own ways and to parallel biomedicine. This process of producing and reproducing power imbalances is hidden not only in the confrontation of knowledge systems but also in TCM physicians' interactions with biomedical doctors and with patients.

\section{Unequal Power Relations in Communication}


Participants observed their low power status in the healthcare system when interacting with biomedical doctors and with patients. Based on their experience, biomedical doctors, particularly younger ones, tend to take all credit from integrative medicine. The interaction pattern infers that many biomedical doctors do not view TCM physicians as equals:

A lot of biomedical doctors think that they are very superior, and they are able to cure patients. If patients see both TCM and biomedical doctors, they would say that they are able to recover because they use both forms of treatment. But biomedical doctors would feel that their credits are halved. A lot of young Western doctors like to snatch credits. Biomedical doctors' superiority also appears in their ability to quickly shift blame to TCM physicians when patients' conditions take a turn for the worse:

There are cases where the patient will complain weeks later that her leg condition starts to worsen. Then the Western practitioners will ask what other medicine did you take, then if the person says acupuncture, normally the Western practitioners will jump to a conclusion that it must be the acupuncture that causes infection to your leg. But it may not be a one- or two-day thing that causes an infection.

With the backing of scientific tools and methods, biomedical doctors are prone to disapprove of TCM physicians' interference in treatments. However, they also often shift the burden of responsibility to TCM physicians when biomedicine has reached an impasse. The observation that biomedical doctors can dictate when TCM is involved in which phase of healing exposes the power differential between biomedical and TCM physicians:

Many Singaporeans trust Western medicine because at least Western medicine can help to control a person's condition and relieve the pain temporarily. But once Western doctors can no longer treat the patient, they will push the responsibility over to TCM 
physicians. And then TCM physicians will try to fix the problem.

TCM physicians found themselves both bearing the blame and playing the role of the last resort. Some participants used the Chinese saying, "trying to rescue the dead horse as if it is alive," to describe their powerless feeling in service provision when multiple trials of biomedical treatments failed to work for the patient. The treatment might not be effective and, subsequently, more criticism could come from biomedical doctors. The vicious circle of treating TCM as a back-up option reproduces the idea that TCM is a supplementary and untrustworthy practice. In this view, participants argued that the so-called integration only sustains biomedical dominance over TCM instead of promoting collaboration based on equality.

Power differentials between biomedicine and TCM can also be identified in TCM physicians' interactions with patients. Allopathic hegemony has created class differences between biomedicine and TCM practitioners, which are reflected in their distinctive salary and socioeconomic status. This implicit exercise of control has further penetrated public perceptions and cultivated an inferior image of TCM. The ideology-loaded prejudice toward TCM can explain some TCM physicians' reporting of patients' attempts to hide their TCM visits in their neighborhood:

I have a few patients who are patients under biomedical specialists. Maybe it is because of societal prejudice, so they come to look for me in a sneaky way. They are afraid of meeting other patients. TCM physicians' salaries cannot be compared with biomedical doctors. Our social status also cannot compete with them. So what do we have in us to combine or integrate with them?

This excerpt reveals that notwithstanding that patients acknowledged the practical value of TCM, their action and attitude were conditioned by the dominant ideology and its narration of TCM. 
The fear of running into acquaintances and being caught by family doctors alludes to acceptance of the dominant view that seeking TCM treatment without preapproval from a biomedical doctor is inappropriate. This perception of TCM as inferior to biomedicine not only nurtures patients' feeling of shame and fear but also shapes the way they interact with TCM practitioners.

Participants observed that their lower power position in the biomedical-dominant system induces a lack of respect from patients and low patient compliance. For instance, patients are more likely to complain about longer waiting times in TCM clinics:

When patients visit Western doctors, they don't dare to say anything, and they are willing to wait up to one or two hours. If they go to a TCM clinic, they always ask, how much longer must I wait? If you tell them they need to wait one more hour, they'll tell you they cannot wait anymore and they have to leave. They just insist on seeing the physician immediately.

TCM physicians are usually perceived as more affable, empathic, and communicative than biomedical doctors. While it is valuable to have a supportive doctor-patient relationship in TCM, the patients' expectation that their requests must be immediately fulfilled and that they can interrupt the diagnostic procedure whenever they want suggests a disparaging view of TCM, which differs distinctly from their view of biomedicine.

The lack of respect was also manifest in patients' tolerance of biomedical failure but being critical toward TCM. Here again, the public's expectation of and response to the two systems resonate with their corresponding power positions in the healthcare system:

If a patient has diabetes, she seeks Western treatment, she takes this medicine, and it is not working. The doctor will say this kind of medication is not suitable for you. We shall switch to another kind of medicine and see how it goes for you. But for TCM, 
patients will say that you are inexperienced, you are not good, your medication is not good because your prescription is not documented.

Participants noted that when biomedical treatment fails to work, patients may want to negotiate alternatives but they do not question biomedical doctors' epistemic authority. In contrast, in circumstances when TCM treatments are ineffective, patients are more likely to place full responsibility on TCM physicians by criticizing their learning modes, epistemic approaches, and the lack of standardization in diagnosis and prescriptions. Biomedical reductionism and objectification have become the universal law for knowledge evaluation across medical systems. Allopathic hegemony cultivates public doubt about TCM's ways of knowing such that whenever TCM treatment is ineffective, the doubt surfaces.

In addition, some participants attributed public deprecation of TCM to the overcrowding presence of charity TCM organizations in Singapore. These physicians argued that charity TCM clinics create the public impression that TCM is of a lower quality and status, leading patients to undervalue the TCM profession and repelling talents from joining the industry:

There are too many free services and they definitely affect the normal business. Would talented people want to be TCM doctors if they can't even earn enough money to cover three meals? So it does affect TCM's standing. If TCM can be more profit-making and have more talents, I believe more people would want to study TCM. And if we can have more talents in TCM, we can develop the field better.

Charity TCM clinics certainly play an important role in preserving TCM practice in local cultural contexts and are important sources of support for the poor and the elderly. However, participants were concerned that low wages and the inferior image of TCM combined with free services may even deepen class differences between biomedicine and TCM and erect more barriers to 
attracting talents. The different social status of TCM and biomedical doctors is a product of biomedical dominance, but this marker may also be used by patients and potential TCM students to form their judgment of TCM, leading to the reproduction of subordination. The ideologically constructed image of TCM and the lack of recognition from biomedical peers and patients coconstruct power imbalances between TCM and biomedicine. The structural force looming in the background that reinforces the power differentials is limited government support.

\section{Interplay of State Support and Allopathic Hegemony}

The interview data revealed that biomedicine maintains its hegemonic presence not only through privileged access to technology and medical discourse but also through the political authority's entitlement. The state's endorsement of biomedical dominance is parallel with its limited recognition of TCM, which is manifest in its conditional support for TCM research, lack of subsidies for TCM treatments, and interference in TCM practice in hospital settings.

Participants noted that since 2012, they can apply for governmental funding for TCM research. However, the condition to use the grant, which demands biomedical doctors' leadership in TCM research, signifies the secondary role of TCM in the government's plan for medical advances and its insufficient support for growing TCM as an independent discipline:

The government has been gradually giving us more support over the years. But of course it can't be compared to the amount of support given to biomedicine. For example, if TCM clinics apply to conduct research, they won't get approved. They must be initiated by a Western doctor. But it's not quite possible, right? Now the requirements have been lax, but still quite impossible for TCM physicians to do research independently. The requirement to include biomedical doctors in a TCM research team, and not vice versa, illustrates how the state aids in the hegemonic control of biomedicine. TCM is relegated to a 
passive role where its development as a holistic practice is restricted.

Likewise, the state has excluded TCM from the national medical insurance scheme. The political authority's allocation of resources resonates with the dominant paradigm's definition and separation of the orthodox and non-orthodox. Participants noted that civil service and most companies in Singapore do not accept medical certificates issued by TCM physicians. Although TCM is many older adults' preferred treatment, its services are not subsidized:

Now Singapore has an aging population, where most of them have very weak Qi and they will feel very tired. A lot of the older generation prefers to go to TCM. But there are no subsidies for TCM. So in terms of healthcare affordability, that is a major obstacle.

With aid from the state, biomedicine is able to sustain its exclusive privileged role in the national healthcare system. In contrast, the cultural importance of TCM and its meaning to local people are omitted during the exclusion process. As ideological differences and unequal social statuses have evoked tensions between the two medical systems, the government's limited support for TCM reinforces the moral and intellectual leadership of biomedicine. The implicit message is that TCM practice is not encouraged; it should be supplementary to biomedicine, rather than a standalone discipline.

The lack of institutional recognition also appears in hospital settings. Participants shared that there were few TCM clinics housed in public hospitals such as the National University Hospital and Tan Tock Seng Hospital. However, in these settings, TCM physicians are merely labeled as acupuncturists even though they hold licenses to practice as full-fledged physicians: Western doctors usually refer patients to go for acupuncture when they feel that acupuncture will not affect their medical treatment. TCM physicians at these clinics can only carry out acupuncture on patients. Even though they are qualified to prescribe 
medicine, they are not allowed to do so. Under such circumstances, it is not up to the patients to decide if they want to see a TCM physician. That is why I feel that TCM becomes very passive in nature, just like a technician.

Participants observed that TCM can only be incorporated into public hospital systems when it is reduced to a subdivision of biomedicine and operates with referral and approval from biomedical doctors. Biomedical doctors hold the power to decide the need for and the use of TCM. This power arises from the state's structural arrangements for service provision and its ability to define the professional boundaries of biomedicine and TCM. However, the state's exercise of political power is interlinked with biomedicine's dominance in medical knowledge. The interplay of these two forces sustains the hierarchical difference between biomedicine and TCM.

Participants shared that no TCM clinics are housed in public clinics, limiting potential dialogue between TCM and biomedicine. While they felt the urge to voice their concerns about the structural settings, they could not find access to the discourse:

If you want to have a TCM clinic in polyclinics, you need to have a really huge department because TCM is a broad category comprising herbal medicine, acupuncture, cupping, etc. Do you think that would happen in the future? I do have seniors who have thought about this too. They hope to enter the government sector and speak out on behalf of us. But so far I think nothing has been pushed through.

Although TCM and biomedicine coexist in Singapore, their interactions at the professional level are limited and asymmetric. TCM clinics are either excluded from the public healthcare system or included only as a subordinate division of biomedicine. Given that the government holds the ultimate power in granting legitimacy to a healing system, the interplay of state support and allopathic hegemony reinforces the hierarchy of power and the different levels 
of autonomy to which each medical model is entitled. As most leaders in charge of regulating the TCM industry hail from biomedical backgrounds, decision making is hardly based on a thorough understanding of the TCM discipline. Our results revealed the importance of including TCM physicians' voice in discourse such that a more balanced and complete view of TCM can be obtained and the reform of the pluralistic medical system of Singapore becomes possible.

\section{Discussion}

The pursuit to combine biomedicine with traditional, alternative, and complementary medicine in healthcare is gaining widespread global attention. Researchers like Airhihenbuwa (1995), however, have also raised concerns about the implementation of integration falling into a "donor-deficit" model, in which traditional practitioners are compelled to "modernize" their practice by accepting biomedical language and logic (p. 57). Biomedicine, then, is not one medical system among others, but a standard to follow (Hollenberg \& Muzzin, 2010). This pattern is observed in the studied context. TCM is marginalized through biomedical dominance at the epistemological level and through the reproduction of power differentials in the form of structural, organizational, and public-perception constraints. The experiences and perceptions shared by TCM physicians expose ideological confrontations underlying the tension between TCM and biomedicine and point out areas where communication can be further enhanced.

Resonating with previous research on medical ideology, this study reveals an infusion of power and control in knowledge systems that endorses a specific group's epistemic authority while mystifying and deprecating the others (Adam et al., 2009; Lupton, 2012). Certainly, TCM differs from biomedicine in a number of respects, including ways to accumulate knowledge, make diagnoses, and suggest treatments. Reductionism and holism reflect different ideological assumptions about health based on which biomedicine and TCM develop different approaches to 
knowledge formation and evaluation. However, this dichotomized framing of medical practices has been extended beyond discussion of ways of knowing to serve the vast interest of biomedical dominance. Dichotomous separation becomes the basis for practicing inclusionary and exclusionary strategies in the healthcare system of Singapore such that a divide is created between the orthodox versus the non-orthodox, the have-access-to-discourse versus the have-not, the have-right-to-use-technology versus the have-no-right, the in-group of national healthcare financial plans versus the out-group, and the leader in decision making and research versus the follower. Extensions of the dichotomized frames of biomedicine vis-à-vis TCM help perpetuate biomedical dominance in two circular steps: first, discrediting and labeling on claims and beliefs that differ from the biomedical standard such that their validity in discursive spaces is weakened and, second, using the misrepresented frames and appraisals to take away resources. Through this dichotomization process, TCM's focus on experiential knowledge is bundled with negative value judgments such as being non-scientific, non-objective, and anti-technology. The negative framing subsequently influences the allocation of resources and enforces the marginalization of TCM. Inasmuch as the extended dichotomous frames continue, TCM practitioners are trapped between two options. They can subscribe to biomedical epistemology to secure resources and retain opportunities for collaboration, but by doing so they accept an inferior position in relation to biomedicine, or they can choose to resist biomedical ideology and use whatever resources remain to provide services and develop the discipline. Either way, the marginalization of TCM is continued.

The expansion of biomedical ideology relies on the exercise of power in both tangible and intangible forms. In this study, ideology and power are mutually reinforcing at three levels: TCM practitioners' relationship with the state, with their biomedical peers, and with patients. 
Structural and organizational constraints demonstrate the more tangible form of power, which involves the political authority's regulation of professional boundaries and its control over resources in service provision and funding. While the state's decision to favor biomedicine may be due to a variety of reasons, including the dominance of biomedicine in global medical discourse and the monopoly of biomedically trained professionals in domestic policymaking, this study reveals the consequences of interflows between ideological and political forces that support power differentials between biomedicine and TCM.

Ideology and power also interact in symbolic and intangible aspects, which involve imposing constraints on discourses, setting norms and expectations, and cultivating dominant values and beliefs. The asymmetric interactions between TCM and biomedical physicians and the different modes of doctor-patient communication in TCM and biomedical practice manifest this exercise of power. State support entitles biomedical doctors to control resources and enjoy higher status in social life and in the workplace. At the intersection of government backing and biomedical dominance in intellectual and moral terms, biomedical doctors can exert intangible power, intentionally or unintentionally, to impose value judgments on TCM physicians and shape public perceptions of the two medical systems such that their privileged status is sustained. Power in this respect is present in biomedical doctors' ability to interpret the suitability and effectiveness of TCM treatments, to omit or disapprove of TCM worldviews and principles in discursive spaces, to treat TCM as a backup plan, and to implement normative pressure on patients' selection of medical treatments. Likewise, patients' interactions with TCM physicians also point toward a social reality of power in which pervasive norms condition the public mentality that biomedicine is the only health practice that is proper and worthy of respect. Power here reflects the control of public perceptions of TCM and involves the permeation of the 
dominant meaning system. This power exercise is apparent in patients' internalization of biomedical standards in guiding their judgment of TCM practice and their own interest in seeking TCM treatment. Ultimately, power and ideology are intertwined to construct the inferiority of TCM. The interplay of the dominant biomedical epistemology, the political forces, and the penetration of hegemonic control at the inter-professional, doctor-patient, and public perception levels fosters inequalities in status between biomedicine and TCM.

\section{Implications}

Inasmuch as TCM remains an integral part of cultural practice and a popular traditional medical practice in Singapore, a critical questioning of the current framings of TCM exposed by its practitioners is helpful for laying out possible pathways for integrative medicine that are more supportive of mutual learning and cooperation. Lupton (1994) suggested that the dominant medical discourse should be critically examined such that the underlying taken-for-granted assumptions can be identified and disrupted. This study illustrates that the dichotomous separation of TCM and biomedicine is saturated with ideological interests and the exercise of power. To change the status quo, more critical discussion of the dichotomized framings should be introduced into discursive spaces. One area for dialogue is rethinking the binary of tradition and technology. The question here is whether a traditional medical system must be confined in the past and not be able to enjoy the benefits of technological advancement and evolve over time. TCM holds a holistic view of health, values traditional knowledge, and relies on physicians' judgment based on years of experience, but following classical principles and using technology to aid diagnosis and treatment need not be at opposite ends of knowledge development. The 2015 Nobel Prize winner in medicine Youyou Tu's (2015) research on artemisinin is an example of how medical innovations can benefit from a coalition of ancient TCM remedies and modern 
laboratory techniques. In the context of Singapore, the restrictions in access to medical instruments have deprived TCM physicians of opportunities to update the discipline and defend the validity of their reasoning system. While different medical systems may present their understanding of health and illness in different ways, technology as an approach to knowledge should not be exclusively owned by one particular paradigm and used to prevent the growth of another. More discussion can be dedicated to this issue in the future.

Political support can be an aid to allopathic hegemony as much as a means of intervening in power differentials and reducing prejudice in society and among health professionals. In recent years, more government interventions have been carried out with a focus on cultivating the education system. For example, the state has moved to set up a TCM discipline in one of the five publicly funded universities in Singapore. The introduction of TCM in university medical education presents an opportunity to create a more amicable environment that welcomes interactions among students in each camp. Although not ideal, growing state support has also been given to opening up spaces for dialogue between medical communities. An example is the Regional Conference on Integrated Care, in which TCM and biomedical doctors were invited to talk on the same platform (Singapore General Hospital, 2014). Building on these initial efforts, more dialogue in medical, political, public, and academic discourses should be encouraged to create a critical awareness of the tensions between TCM and biomedicine and the underlying reasons. Such awareness, together with a balanced view of different medical practices, is key to the development of a more collaborative and communicative healthcare system in Singapore.

With respect to limitations, in this study we interviewed TCM physicians with different demographics to maximize variation in their accounts. During data analysis, we observed a generational difference in participants' attitudes toward further collaboration between 
biomedicine and TCM. Younger physicians believed in the merits of both disciplines, acknowledged limitations inherent within each discipline, and advocated the use of biomedicine when necessary. In contrast, older physicians showed stronger opposition toward biomedicine and insisted that TCM is the best option. This generational difference did not affect the extracting of themes commonly shared by all participants. However, in the future, researchers can consider comparing TCM physicians in different age groups who may vary in their perception of, and reaction to, the dynamics between TCM and biomedicine. After including TCM physicians' voice in discourse, it may also be helpful to explore biomedical doctors' points of view and extract additional insights regarding possible directions for integrative medicine.

\section{Conclusion}

This study centralizes TCM physicians' voice to unveil the source and site of ideological struggles and power differentials that foster the marginalization of TCM. Knowledge, power, the state, and social interactions are interlinked components of a hierarchical differentiation between dominant and marginalized medical practices. Given their distinct philosophical and epistemological views of health, divergences in medical concepts and reasoning systems might be unavoidable for TCM and biomedicine. Nevertheless, more open dialogue should be encouraged to recognize these differences. The key to reducing tensions lies in developing a more critical awareness of the power-loaded dichotomous framings of biomedicine vis-à-vis traditional medicine, as well as in identifying common grounds for health professionals to interact and initiate dialogue. Ultimately, the goal is to achieve a more supportive system of medical pluralism that encourages, instead of confines, growth in all medical paradigms and provides better healthcare services to those in need of them. 


\section{References}

Adams, J., Hollenberg, D., Lui, C., \& Broom, A. (2009). Contextualizing integration: A critical social science approach to integrative health care. Journal of Manipulative and Physiological Therapeutics, 32, 792-798.

Airhihenbuwa, C. O. (1995). Health and culture: Beyond the Western paradigm. Thousand Oaks, CA: Sage.

Barry, C. A. (2006). The role of evidence in alternative medicine: Contrasting biomedical and anthropological approaches. Social Science \& Medicine, 62, 2646-2657.

Basnyat, I. (2017). Theorizing the relationship between gender and health through a case study of Nepalese street-based female sex workers. Communication Theory. Advance online publication. doi: 10.1111/comt.12114

Basu, A., \& Dutta-Bergman, M. (2007). Centralizing context and culture in the co-construction of health: Localizing and vocalizing health meanings in rural India. Health Communication, 21, 187-197.

Braun, V., \& Clarke, V. (2013). Successful qualitative research: A practical guide for beginners. London, UK: Sage.

Chang, L., \& Basnyat, I. (2015). Negotiating biomedical and traditional Chinese medicine treatments among elderly Chinese Singaporean women. Qualitative Health Research, 25, 241-252.

Dutta, M. J. (2008). Communicating health: A culture-centered approach. London, UK: Polity Press.

Eakin, J., Robertson, A., Poland, B., Coburn, D., \& Edwards, R. (1996). Towards a critical social science perspective on health promotion research. Health Promotion International, 11, 
157-165.

Elling, R. H. (1981). Political economy, cultural hegemony, and mixes of traditional and modern medicine. Social Science \& Medicine, 15A, 89-99.

Filc, D. (2004). The medical text: Between biomedicine and hegemony. Social Science \& Medicine, 59, 1275-1285.

Hollenberg, D. (2006). Uncharted ground: Patterns of professional interaction among complementary/alternative and biomedical practitioners in integrative health care settings. Social Science \& Medicine, 62, 731-744.

Hollenberg, D., \& Muzzin, L. (2010). Epistemological challenges to integrative medicine: An anti-colonial perspective on the combination of complementary/alternative medicine with biomedicine. Health Sociology Review, 19, 34-56.

Hu, D. (2016). Traditional Chinese medicine: Theory and principles. Beijing, China: Tsinghua University Press.

Keshet, Y., \& Giveon, A. (2013). Integrative health care in Israel and traditional Arab herbal medicine: When health care interfaces with culture and politics. Medical Anthropology Quarterly, 27, 368-384.

Khan, S. (2006). Systems of medicine and nationalist discourse in India: Towards "new horizons" in medical anthropology and history. Social Science \& Medicine, 62, 27862797.

Khan, S. (2014). Manufacturing consent? Media messages in the mobilization against HIV/AIDS in India and lessons for health communication. Health Communication, 29, 288-298.

Koh, H., Ng, H., \& Teo, H. (2004). A survey on knowledge, attitudes and usage of complementary and alternative medicine in Singapore. Asia-Pacific Biotech News, 8, 
1266-1270.

Ku, H. Y., Tan, M., \& Li, S. C. (2001). Attitude and knowledge towards traditional medicine among Singaporeans. Abstract book of the Singapore Traditional Chinese Medicine Research Symposium. Singapore, 40-41.

Lambert, H. (2012). Medical pluralism and medical marginality: Bone doctors and the selective legitimation of therapeutic expertise in India. Social Science \& Medicine, 74, 1029-1036.

Lee, T. (2006). Complementary and alternative medicine, and traditional Chinese medicine:

Time for critical engagement. Annals of the Academy of Medicine, Singapore, 35, 749750.

Lim, M. K., Sadarangani, P., Chan, H. L., \& Heng, J. Y. (2005). Complementary and alternative medicine use in multiracial Singapore. Complementary Therapies in Medicine, 13, 16-24.

Lukes, S. (2005). Power: A radical view (2 ${ }^{\text {nd }}$ edition). Basingstoke, UK: Palgrave.

Lupton, D. (1994). Toward the development of critical health communication praxis. Health Communication, 6, 55-67.

Lupton, D. (2012). Medicine as culture: Illness, disease and the body. Los Angeles, CA: Sage.

Ministry of Health Singapore. (2010). Regulation of traditional Chinese medicine (TCM). Retrieved from http://www.moh.gov.sg/content/moh_web/moh_corp_mobile/home/our_healthcare_syste $\underline{\mathrm{m} / \text { healthcareregulation/regulationoftcm.html }}$

Ministry of Health Singapore. (2014). TCM practitioners. Retrieved from https://www.moh.gov.sg/content/moh_web/healthprofessionalsportal/tcmpractitioners.ht $\underline{\mathrm{ml}}$

Quah, S. R. (2003). Traditional healing systems and the ethos of science. Social Science \& 
Medicine, 57, 1997-2012.

Russo, E., Ania, F., \& Crellin, J. (2012). Professionalism and ethics in complementary and alternative medicine. New York, NY: Routledge.

Schreiber, L. (2005). The importance of precision in language: Communication research and (socalled) alternative medicine. Health Communication, 17, 173-190.

Singapore General Hospital. (2014, Aug 11). Regional Conference on Integrated Care

Programme. Retrieved from http://www.sgh.com.sg/clinical-departments-

centers/nursing/rciac/pages/programme.aspx

Tu, Y. (2015). Nobel Lecture. Retrieved from

https://www.nobelprize.org/nobel_prizes/medicine/laureates/2015/tu-lecture.html

Wiese, M., Oster, C., \& Pincombe, J. (2010). Understanding the emerging relationship between complementary medicine and mainstream health care: A review of the literature. Health, 14, 326-342.

World Health Organization. (2008). Beijing Declaration. Retrieved from http://www.who.int/entity/medicines/areas/traditional/TRM_BeijingDeclarationEN.pdf

Zoller, H. M., \& Kline, K. N. (2008). Theoretical contributions of interpretive and critical research in health communication. Communication Yearbook, 32, 89-136. 